\title{
Combined VLT ISAAC/ISO SWS spectroscopy of two protostellar sources
}

\section{The importance of minor solid state features}

\author{
E. Dartois ${ }^{1}$, L. d'Hendecourt ${ }^{1}$, W. Thi ${ }^{2,3}$, K. M. Pontoppidan ${ }^{3}$, and E. F. van Dishoeck ${ }^{3}$ \\ 1 “Astrochimie Expérimentale”, IAS-CNRS, Bât. 121, Université Paris Sud, 91405 Orsay Cedex, France \\ 2 Department of Physics and Astronomy, Univeristy College London, WC1E 6BT, UK \\ 3 Sterrewacht Leiden, PO Box 9513, 2300 RA Leiden, Netherlands *
}

Received 27 May 2002 / Accepted 26 August 2002

\begin{abstract}
We discuss the $L$ band infrared spectra of two massive protostars in the region of the so-called $3.47 \mu \mathrm{m}$ absorption feature. The high sensitivity VLT-ISAAC spectra of the two differently evolved massive protostars GL 989 and GL 2136 , together with dedicated infrared laboratory experiments on ice molecular interactions, allow us to propose the identification of this feature with the formation of an ammonia hydrate. Combined with an analysis of additional ISO observations, the derived ratio of solid $\mathrm{NH}_{3} / \mathrm{H}_{2} \mathrm{O}$ present in ice mantles is less than or equal to $7 \%$. This amount fits well with the nitrogen cosmic abundance and the chemical evolution of ices where other nitrogen containing $\mathrm{OCN}^{-}$and $\mathrm{NH}_{4}^{+}$ions are observed.
\end{abstract}

Key words. ISM: individual objects: GL 2136, GL 989 - stars: formation - astrochemisty

\section{Introduction}

The presence of physisorbed ices covering the refractory interstellar grains in dense regions has been detected more than two decades ago (e.g. Capps et al. 1978; Willner et al. 1982; Merrill et al. 1976), mainly through the observation of the strong absorption from the $\mathrm{OH}$ stretching vibration of water ice at $\sim 3.05 \mu \mathrm{m}$, later followed by the first detection of the solid CO absorption at $4.62 \mu \mathrm{m}$ (Soifer et al. 1979).

From the start, these observations were confronted with laboratory simulations which provide a unique and accurate tool to identify ice species, the composition of which were first "guessed at", following some models of gas-grain chemical interactions (Tielens \& Hagen 1982; d'Hendecourt et al. 1985).

It appeared quickly that the study of line profiles is of prime importance in the identification of solid state species, as shown early by Léger et al. (1979), demonstrating the radical change of the $\mathrm{OH}$ stretching mode absorption upon evolution from the amorphous to crystalline state of the ice. In the mid-infrared range, spectra provided by the Infrared Space Observatory

Send offprint requests to: E. Dartois,

e-mail: emmanuel.dartois@ias.u-psud.fr

* Based on observations collected at the European Southern Observatory, Chile (ESO 164.I-0605(A)). ISO is an ESA project with instruments funded by ESA Member States (especially the PI countries: France Germany, The Netherlands and the UK) and with the participation of ISAS and NASA. allow an estimate of the global composition of the dominant ices in many sources (d'Hendecourt et al. 1996; Whittet et al. 1996; Gibb et al. 2001). However, for deeply embedded sources with low fluxes, e.g. at short wavelengths, the need for ground based large telescopes, such as the Very Large Telescope (VLT) of the European Southern Observatory (ESO), becomes crucial to reach enough signal-to-noise for the interpretation of the spectra (van Dishoeck et al. 2002).

Infrared absorption spectroscopy suffers from a contrast limitation, which implies for the ice constituents that, as opposed to radio observations, the dynamic range is severely restricted. Except for very high oscillator strength transitions (e.g. $\mathrm{OCS}, \mathrm{OCN}^{-}$), it is hardly possible to detect a species whose abundance relative to the water ice falls below the $1 \%$ level. Moreover, the overlap of very deep absorption features such as those of water ice mantles and silicate cores also inhibit the observation of many fingerprints of minor species.

Understanding the composition and structure of ice mantles is therefore a complex interplay between using high signalto-noise spectra often in a quite restricted wavelength range such as that obtained from VLT-ISAAC observations, a wide range spectroscopic coverage, and a strict and careful comparison with laboratory measurements. This latter approach using well-known matrix isolation techniques is particularly appropriate to study the effect of molecular interactions within ice constituents (e.g. the formation of molecular complexes), which allow in turn an accurate understanding of band shapes 
and line widths, revealing the physical and chemical nature of the ice (Ehrenfreund et al. 1998; Dartois et al. 1999).

The composition deduced in some features must also satisfy the fit over a very large wavelength range, and a coherent interpretation must take into account, when available, a combination of ground and space-based observations.

From the astrophysical point of view we investigate in this paper the global spectrum of two young and massive embedded sources at different stages in the evolution of the grain mantles. We associate the so-called " 3.47 " $\mu \mathrm{m}$ absorption band with the appearance of an hydrate containing ammonia. The occurrence of this feature in the spectra of embedded young stellar objects is known since the studies of Allamandola et al. (1992), who attributed this feature to diamond-like material. Brooke et al. $(1996,1999)$ showed that this feature correlates with water ice, suggesting that the species responsible for this absorption is more volatile and linked to the ice mantle. Our present explanation for the " 3.47 " $\mu \mathrm{m}$ feature leads to an estimate of the observed nitrogen content in the ices, satisfying the typical cosmic elemental abundances. We also present a laboratory analysis to demonstrate why the strongest modes of ammonia and methanol, around $9-10 \mu \mathrm{m}$, are difficult to extract from interstellar spectra.

Our observations are reported in Sect. 2. We then describe the experiments performed in order to interpret these data in Sect. 3. We present the results in Sect. 4 and discuss the influence of the scattering on the observed $3 \mu \mathrm{m}$ ice profile. We then analyze the weaker $3.47 \mu \mathrm{m}$ feature from the laboratory point of view, followed by a discussion of this absorption. The implication for other regions of the infrared spectrum from the laboratory results is adressed by examining the global spectrum obtained by combining the ISO (especially focusing on the $9 \mu \mathrm{m}$ region) and VLT data. Finally we discuss the astrophysical issues.

\section{Observations}

The high signal-to-noise observations of GL 989 and GL 2136 sources were obtained at mount Paranal, using the ISAAC instrument on VLT-UT1 (ANTU) in Chile. Both $L$ and $M$ band spectra were taken. We focus here on the $L$ band spectroscopy. The ISAAC mid-infrared spectrometer was used with a grating slit of $0.3^{\prime \prime}$ to $0.6^{\prime \prime}$, low and medium resolution modes, corresponding to $\Delta \lambda / \lambda$ of $\sim 600$ to 6700 in the $L$ band. With the low resolution mode, the entire $L$ band is recorded within a single setting, avoiding the need to stitch parts of the spectra together.

The telluric and background noise was removed using the so-called "chopping-nodding" technique, widely applied in thermal infrared spectroscopy. A reference star, whose absolute flux is known, was observed immediately before or after the science target, within an airmass difference of less than 0.015 . This reference was used to both cancel the telluric absorption arising from the atmospheric transmission and calibrate the flux scale of the science target. We also observed the spectrum of the AGB star $\mathrm{OH} 231.8+4.2$ to provide a pure crystalline ice spectroscopic standard to compare with GL 2136. We discuss in more details this approach in a following section.
In order to get additional information on the observed targets, we also retrieved the Short Wavelength Spectra (SWS) from the Infrared Space Observatory (ISO) of the above two sources, observed on 11/01/97 (GL 989) and 10/11/96 (GL 2136). These spectra were reduced using the Interactive Analysis software installed at IAS and using pipeline processing version OLP10 (de Graauw et al. 1996). Care was taken to separate the upward and downward scans of the spectrometer to check the reproducibility of the detector response, and eliminate spurious features that do not appear in both "up" and "down" scans.

\section{Experiments}

Experiments were conducted in the laboratory at the Institut d'Astrophysique Spatiale to produce ice spectra in order to interpret the observations. Gases were condensed from a deposition line at ambient temperature on a cold CsI window transmitting in the infrared. The window is kept at $10 \mathrm{~K}$ in a cryogenic cell during the experiments by a liquid helium flow cryostat. Infrared spectra are recorded using a Bruker FTS IFS$66 \mathrm{v}$, with a resolution of $1 \mathrm{~cm}^{-1}$. Such matrix isolation spectroscopy and its application to astronomical issues is abundantly described in the literature (e.g. Allamandola et al. 1988; d'Hendecourt \& Dartois 2001).

\section{Results}

The spectra of the GL 989 and GL 2136 sources are presented in Fig. 1, where our VLT observations are combined with the available ISO spectra of these sources. Major solid state features are clearly apparent in the spectra and labeled above each spectrum.

Both sources are massive protostellar objects, GL 989 presenting less evolved ices than GL 2136 as evidenced by the $3 \mu \mathrm{m}$ ice band. The term "evolved" is discussed below. They belong to the same class of high luminosity deeply embedded objects $\left(7.2 \times 10^{4} L_{\odot}\right.$ for GL 2136 , Kastner et al. 1994; $3.3 \times 10^{3} L_{\odot}$ for GL 989 , Henning et al. 1990).

\subsection{Main features: Differences in the ice composition}

In a first step we list the obvious features present in the two studied sources in Table 1 and labeled in Fig. 1. The special cases of the so-called " $3.47 \mu \mathrm{m}$ " and the methanol band at $3.53 \mu \mathrm{m}$ will be discussed later. This table clearly shows that contrary to what is found in GL 2136, the $4.62 \mu \mathrm{m}$ "OCN" " feature is absent in GL 989, or at least one order of magnitude less abundant as compared to water ice. In addition, GL 989 has a solid $\mathrm{CO}$ to $\mathrm{H}_{2} \mathrm{O}$ ratio three times higher than the more evolved source GL 2136. Together with the high degree of $\mathrm{H}_{2} \mathrm{O}$ crystallinity encountered in GL 2136, as evidenced by the shape of the $3 \mu \mathrm{m}$ band, we can safely state that we are comparing similar sources at two different stages of evolution, with the latter source already subjected to partial desorption and/or chemical and thermal evolution. 

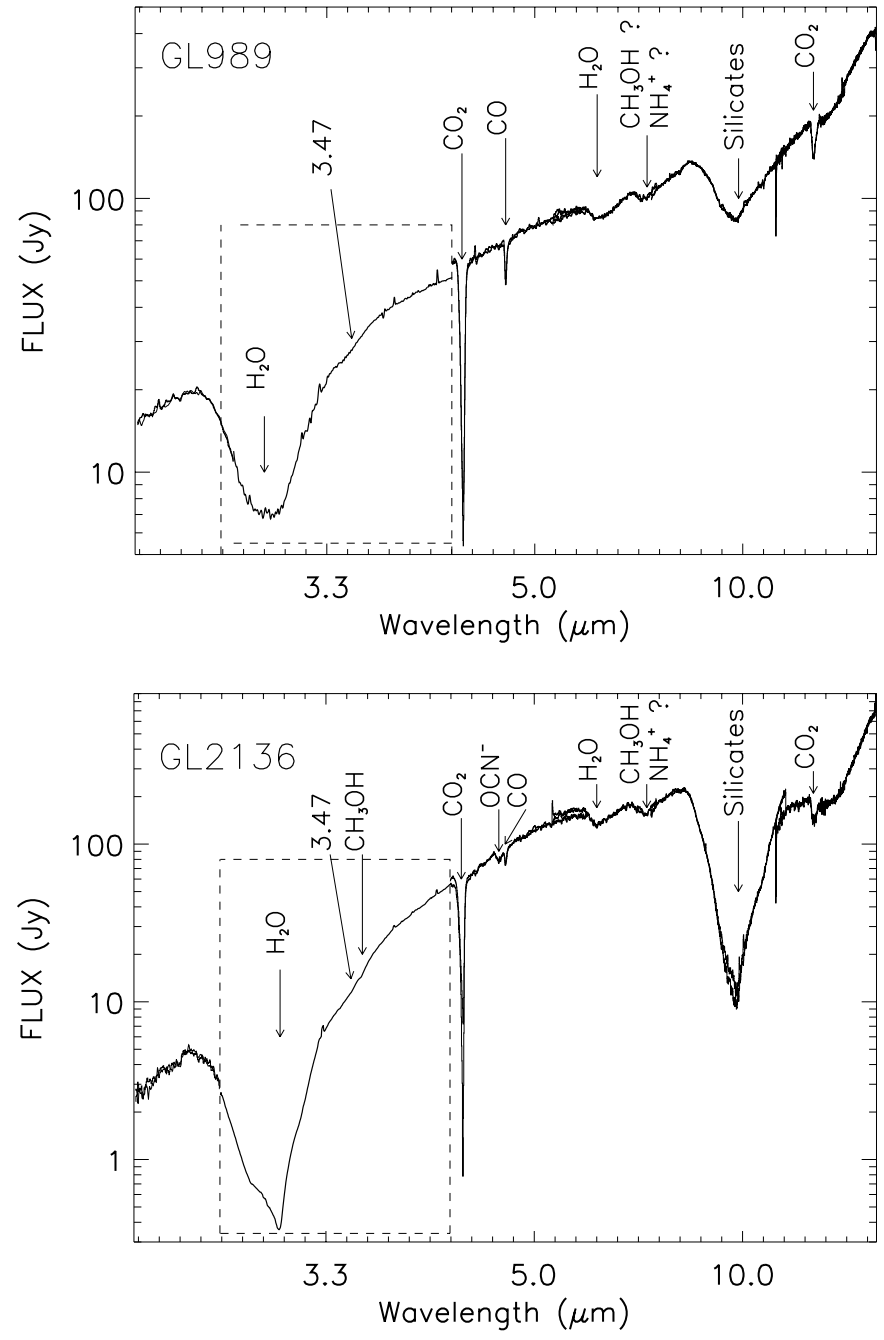

Fig. 1. VLT-ISO combined spectra of the massive protostellar objects GL 989 and GL 2136. The dashed insert box represent the VLT observations in $L$ band. ISO upward and downward SWS scans are overplotted, in order to estimate systematic errors such as memory effects in the detectors. No stitching factor was applied to these data, nor corrections for different apertures.

\subsection{Weaker ice features}

In order to investigate the presence of additional absorption features to the strong water ice $\mathrm{OH}$ stretching vibrations in the VLT data, we extract a local continuum in the region around $3.5 \mu \mathrm{m}$ (Figs. 2 and 3). These extractions reveal the presence of a relatively sharp absorption feature arising at $\sim 3.54 \mu \mathrm{m}$, attributable to the methanol $v_{3} \mathrm{CH}$ stretching mode (Allamandola et al. 1992; Dartois et al. 1999).

Both sources also exhibit a broad absorption at " $3.47 \mu \mathrm{m}$ ", a feature widely observed in our VLT sample of objects observed with the VLT-ISAAC spectrometer (Thi et al. 2002). In the case of GL 989, we observe this absorption feature with the low $(\sim 600)$ and medium $(\sim 6700)$ resolution modes. This allows us to check for the true underlying profile of this band. The medium resolution observations demonstrate that this band is intrinsically structureless, and possesses the same optical depth
Table 1. Prominent features.

\begin{tabular}{cccc}
\hline \hline Feature/molecule & GL2136 & GL989 & \\
& Column $^{\mathrm{a}}$ & Column $^{\mathrm{a}}$ & $\mathrm{A}^{\mathrm{b}}$ \\
\hline $3.1 \mu \mathrm{m} / \mathrm{H}_{2} \mathrm{O}$ & $63 \pm 9$ & $32 \pm 6$ & 20 \\
$4.62 \mu \mathrm{m} / \mathrm{OCN}^{-}$ & $0.24 \pm 0.04$ & $<0.03$ & 43 \\
$4.67 \mu \mathrm{m} / \mathrm{CO}$ & $2.1 \pm 0.6$ & $3.4 \pm 0.8$ & 1.1 \\
$10 \mu \mathrm{m} / \mathrm{Silicates}$ & $47 \pm 5$ & $9.5 \pm 2$ & $16^{\mathrm{c}}$ \\
$15.2 \mu \mathrm{m} / \mathrm{CO}_{2}$ & $5.3 \pm 0.9$ & $5.2 \pm 0.9$ & 1.1 \\
\hline
\end{tabular}

a Column density in units of $10^{17} \mathrm{~cm}^{-2}$, assuming the $A$ values mentioned in the last column of the table.

b $A$ values adopted in units of $10^{-17} \mathrm{~cm} \mathrm{~mol}^{-1}$. For comparison with column density estimates based on other laboratory data (e.g. for $\mathrm{OCN}^{-}$), the $A$-values should be adjusted accordingly.

c This value for the silicates was estimated using a laboratory spectrum of a typical pyroxene containing $30 \%$ of iron, and is provided for inter-comparison between sources.
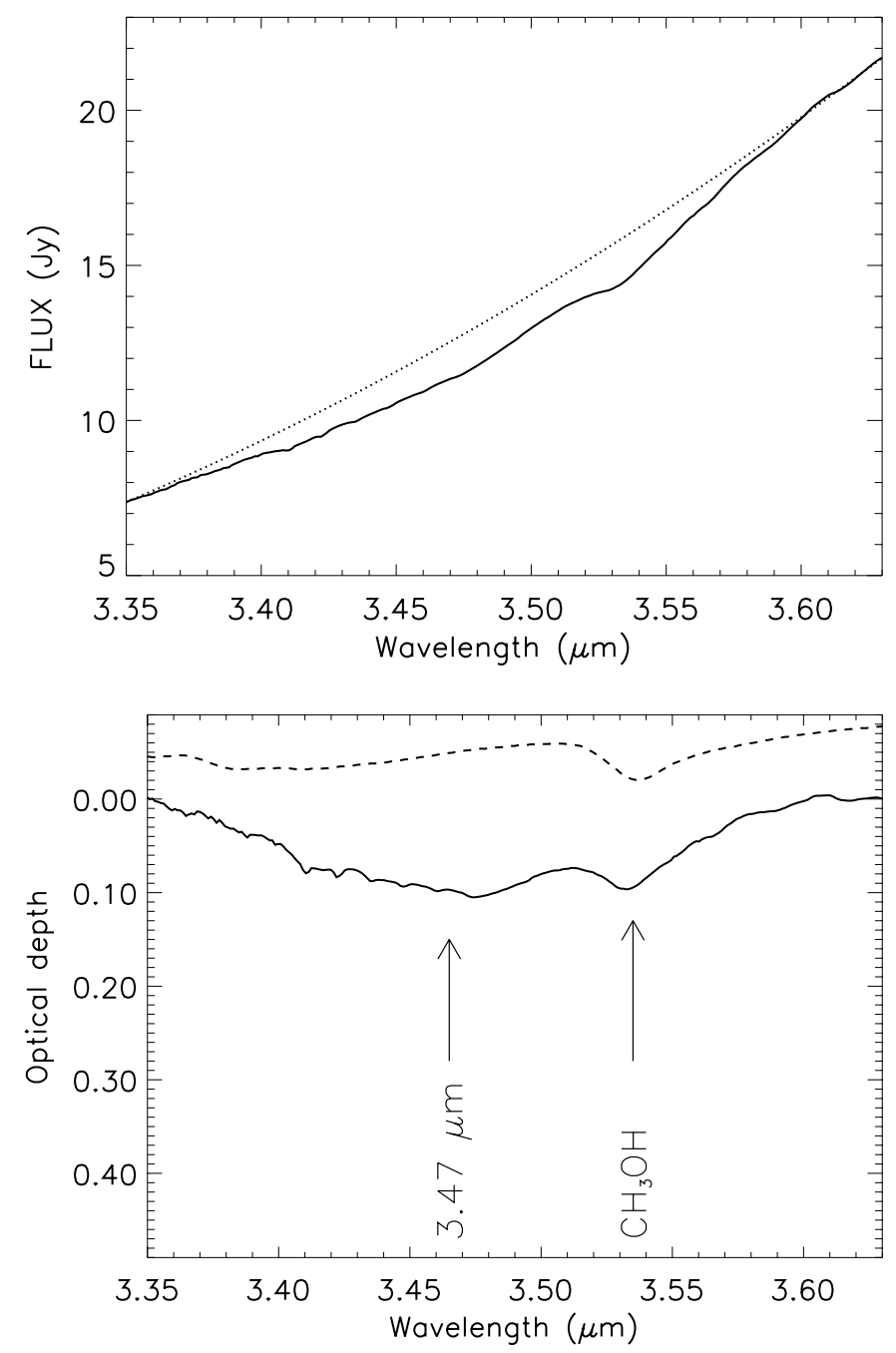

Fig. 2. Upper panel: medium resolution ( 6700) VLT-ISAAC spectrum of GL 2136 around the $3.47 \mu \mathrm{m}$ absorption feature and adopted local continuum (dotted line). Lower panel: resultant optical depth spectrum and over-plotted laboratory pure $\mathrm{CH}_{3} \mathrm{OH}$ ice spectrum (dashed line). 

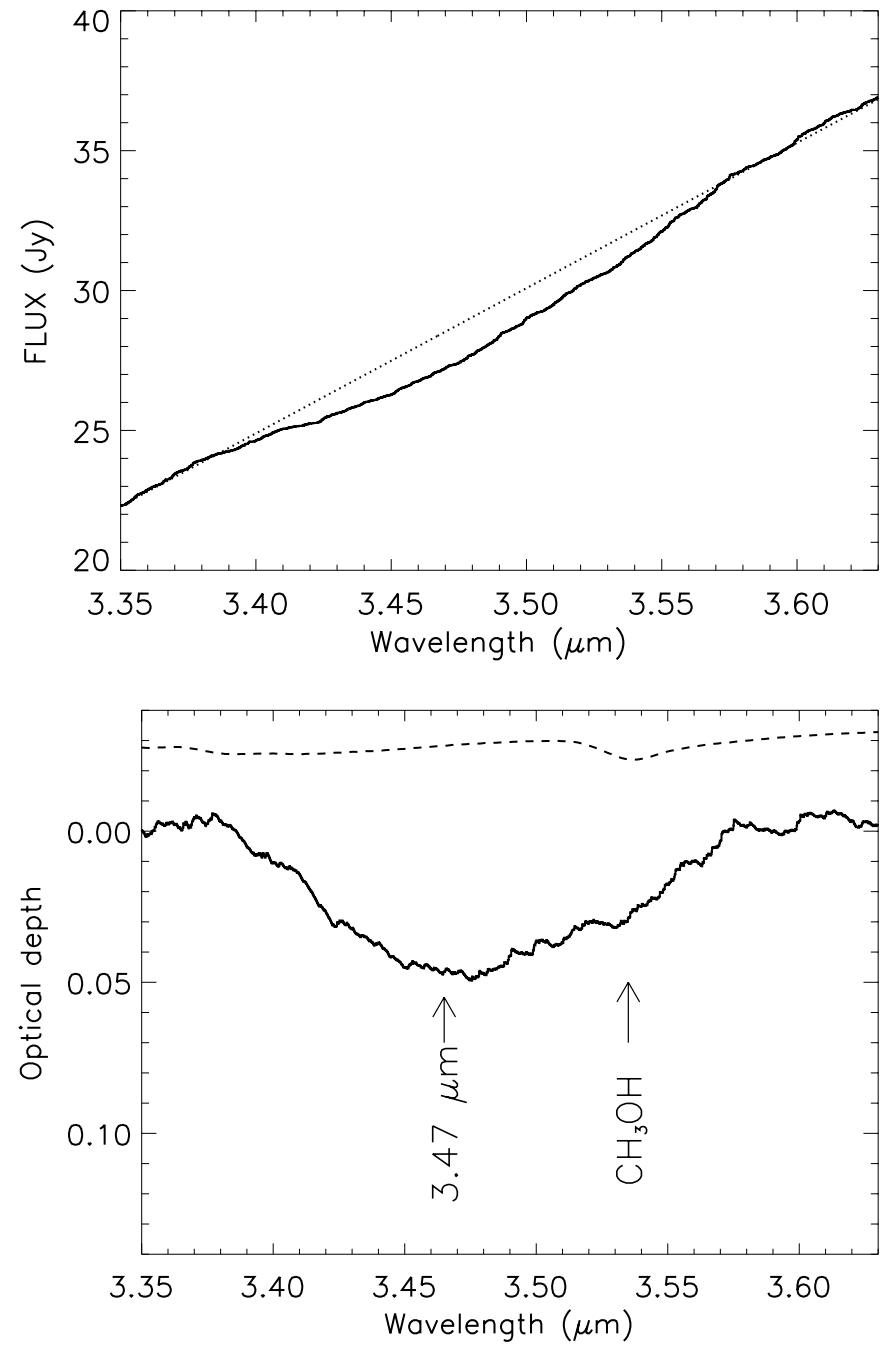

Fig. 3. Upper panel: low resolution $(<600)$ smoothed VLT-ISAAC spectrum of GL 989 around the $3.47 \mu \mathrm{m}$ absorption feature and adopted local continuum (dotted line). Lower panel: resultant optical depth spectrum and over-plotted laboratory pure $\mathrm{CH}_{3} \mathrm{OH}$ ice spectrum (dashed line).

at the two resolutions used. Therefore, it indicates that it is neither due to gas phase absorption lines nor classical aliphatic absorption, such as that observed toward the Galactic center, where a peculiar $\mathrm{CH}_{2}-\mathrm{CH}_{3}$ four peak substructure is expected (e.g. Pendleton et al. 1994; Sandford et al. 1991).

\section{5. $3.47 \mu \mathrm{m}$ mode versus scattering}

The local continuum extraction is a widely used technique to estimate the optical depth of an absorption feature in astronomical spectra. This approach is generally valid for gas phase lines or relatively sharp solid-state features. In the case of the broad $3.47 \mu \mathrm{m}$ mode, the underlying local continuum has to be justified. In particular it is important to check whether part of the optical depth at this wavelength can be attributed to scattering occurring in the wing of the water ice absorption, an effect linked to the grain sizes. To be able to make such a comparison without introducing any modeling of these scattering effects, we have observed the water ice absorption in the late-type star

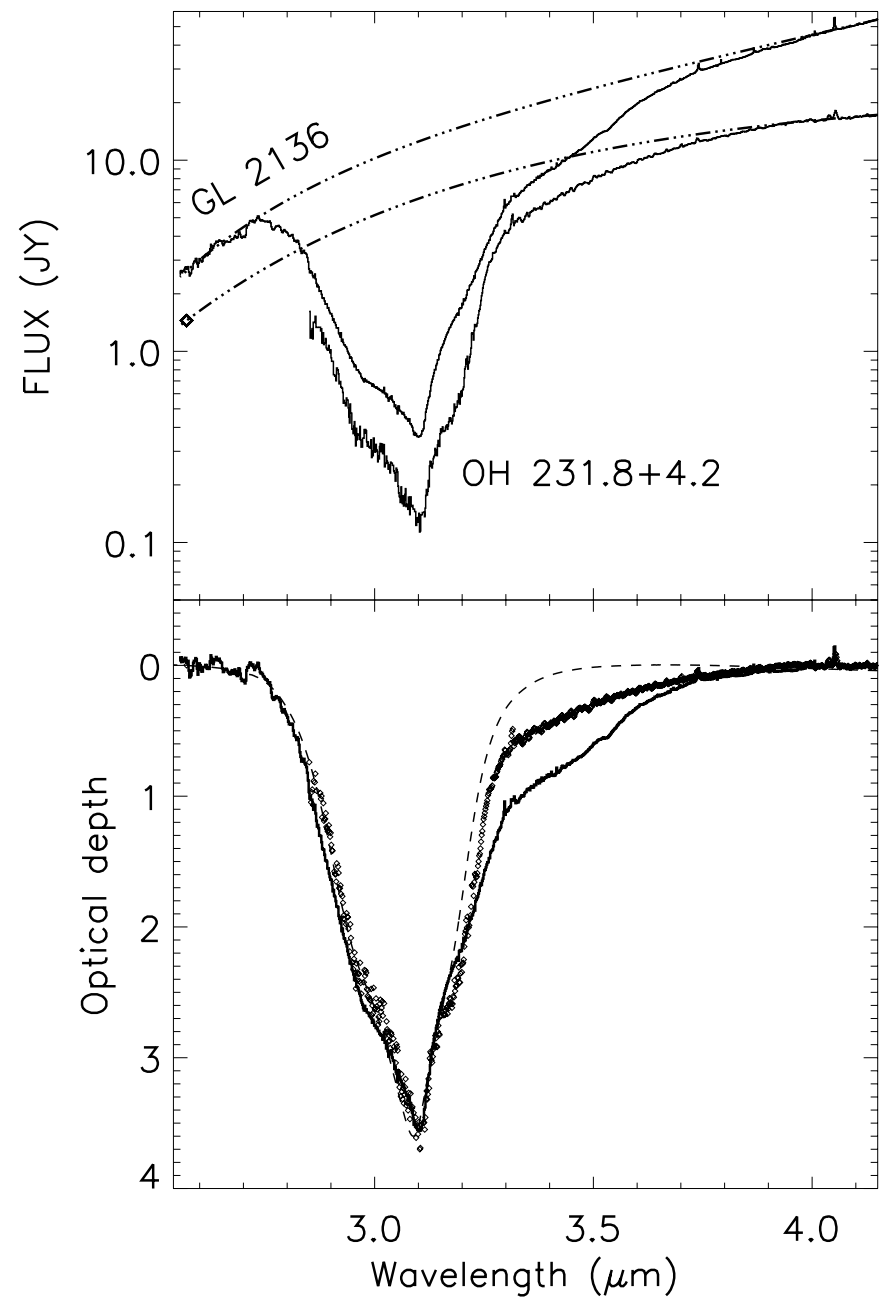

Fig. 4. Comparison between GL 2136 and $\mathrm{OH} 231.8+4.2$ spectra measured with the ISAAC-VLT spectrometer between 2.85 and $4.15 \mu \mathrm{m}$. Below $2.85 \mu \mathrm{m}$, additional data from ISO were added to the GL 2136 spectrum and a $K$ band point from Smith et al. 1988 for the $\mathrm{OH}$ source. Upper panel: fluxes and adopted continua. Lower panel: optical depth spectra for the water ice absorption as measured in laboratory thin films (dashed line), the $\mathrm{OH} 231.8+4.2$ depth (diamond) and the GL 2136 depth. All optical depths are normalized to GL 2136.

$\mathrm{OH} 231.8+4.2$, a totally different environment than that encountered in young stellar objects such as GL 2136. In these $\mathrm{OH}-\mathrm{IR}$ environments, it is known that the only ice features observed correspond to pure water ice (e.g. Roche \& Aitken 1984), generally in a crystalline state as the $\mathrm{H}_{2} \mathrm{O}$ molecules are condensed out from a relatively hot gas phase on grains warmer than those encountered in dark clouds. Such an observation provides a sort of direct "astronomical spectroscopic standard" for the water ice, reproducing similar effects in the wing of the water ice absorption if the grain size distributions are to first order equivalent.

We display the comparison between GL 2136 and the $\mathrm{OH} 231.8+4.2$ "spectroscopic standard" in Fig. 4. The upper panel presents the global continuum extraction in both sources using the ISAAC-VLT spectra from 2.85 to $4.15 \mu \mathrm{m}$, combined with the ISO spectrum at shorter wavelength for GL 2136 and the reddest point in the $K$ band part of the $\mathrm{OH} 231.8+4.2$ 
Table 2. Models parameters.

\begin{tabular}{lrr}
\hline \hline & GL 989 & GL 2136 \\
\hline$a_{\min }(\mu \mathrm{m})$ & 0.01 & 0.01 \\
$a_{\max }(\mu \mathrm{m})$ & 0.75 & 0.75 \\
mantle/core volume ratio & 3 & 3 \\
\hline pure water ice mantle composition & $a$ & $a$ \\
\hline $\mathrm{H}_{2} \mathrm{O} 15 \mathrm{~K}$ & $56 \%$ & $39 \%$ \\
$\mathrm{H}_{2} \mathrm{O} 30 \mathrm{~K}$ & $-\%$ & $23 \%$ \\
$\mathrm{H}_{2} \mathrm{O} 80 \mathrm{~K}$ & $33 \%$ & $-\%$ \\
$\mathrm{H}_{2} \mathrm{O} 160 \mathrm{~K}$ & $11 \%$ & $38 \%$ \\
\hline
\end{tabular}

${ }^{\text {a }}$ Percentage are by volume.

spectrum from Smith et al. (1988). The lower panel displays the optical depths of the water ice absorption as measured in laboratory thin films (dashed line), the $\mathrm{OH} 231.8+4.2$ depth (diamonds) and the GL 2136 depth. All optical depths are normalized to that of GL 2136.

The comparison between the laboratory ice film and the $\mathrm{OH}-\mathrm{IR}$ source shows that there is an excess in the water ice wing, due to the presence of scattering effects. The comparison between the OH-IR source and GL 2136 explicitly demonstrates that some additional absorption remains in the GL 2136 ice absorption wing. Part of this excess may be explained by the bigger grain sizes expected in the latter source, providing additional scattering. However, the scattering cannot explain the sudden change in slope beginning at around $3.3 \mu \mathrm{m}$, due to the presence of additional absorption by the methanol molecule and the species responsible for the $3.47 \mu \mathrm{m}$ band.

\section{Ice profile modeling}

After this preliminary comparison that shows some scattering exists in the water ice wing, we model the expected optical depth of the $3 \mu \mathrm{m}$ ice absorption using Mie theory, for an ice mantle covering a silicate core. We take into account different water ice temperatures as well as the contribution from methanol. This procedure is described elsewhere (Dartois \& d'Hendecourt 2001). For the silicates we used the Draine \& Lee (1984) optical constants. We implement also a grain size distribution for the radii of particles. We choose the classical MRN (Mathis et al. 1977) power law distribution, with a lower grain radius size boundary of $a_{\min }=100 \AA$ and a variable upper size $a_{\max }$. We extract the ice profile by fitting a local continuum in the resultant model extinction, on each side of the water ice mode, to reproduce the extraction process in astronomical spectra. Parameters used for this modeling for each source are summarized in Table 2. Note that we did not take into account a possible onion like ice structure versus spatially segregated different mantles (Ehrenfreund et al. 1998), nor additional contributions from hydrocarbons or PAHs.

Given the large number of parameters involved in such an approach (ice temperatures involved, exact nature of the silicates, power law index, volume ratio between the core and mantle, value of $a_{\max }$ ), the resultant profile obtained in this approach is only intended to obtain a realistic ice optical depth
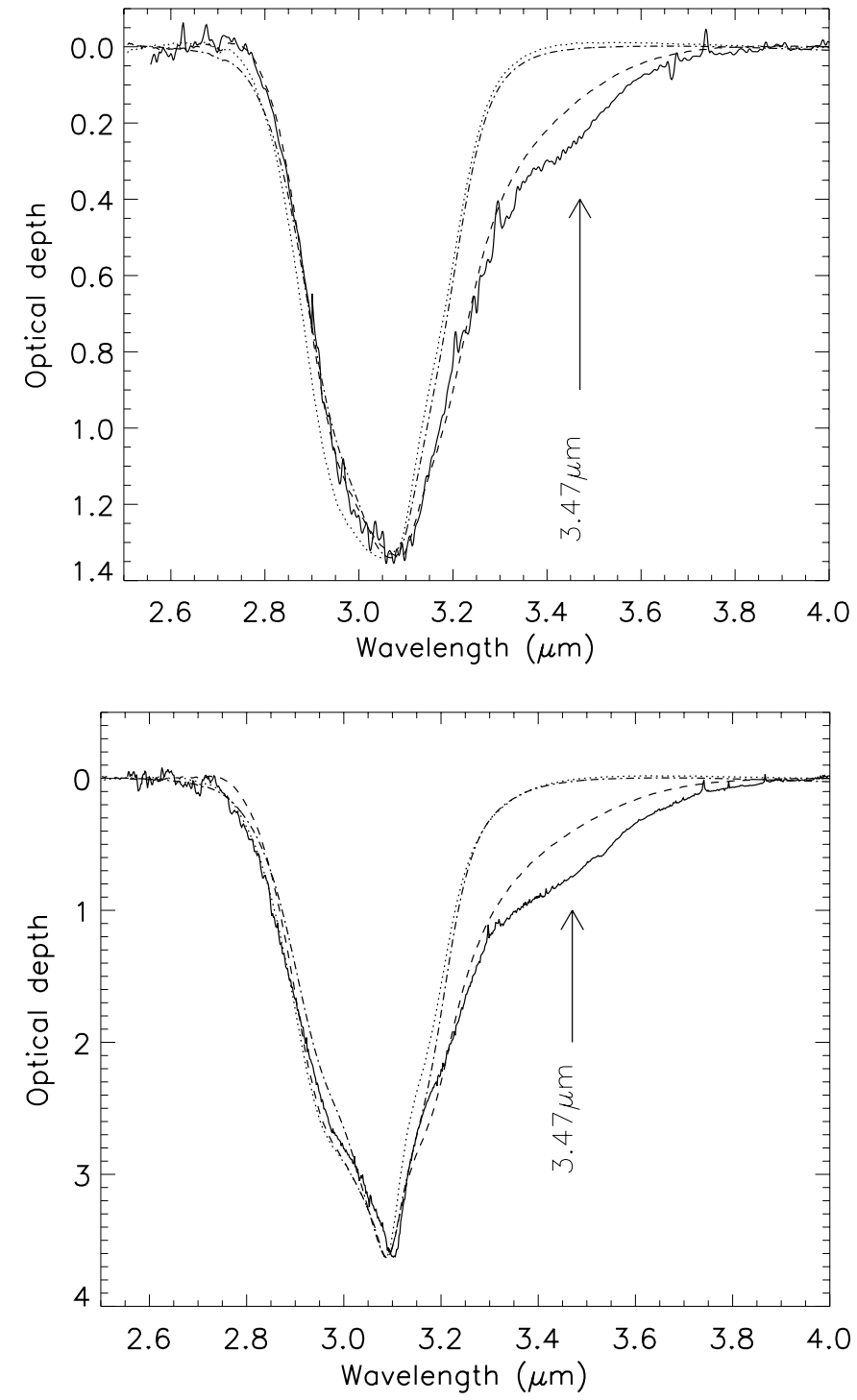

Fig. 5. Optical depth spectra of GL 989 (upper panel) and GL 2136 (lower panel) in the $L$ band. The dashed line represents the silicate core-ice mantle fit using laboratory data and parameters described in Table 2. For each panel, the dotted line is the extinction by a core-ice mantle for a grain that is small compared to the wavelength. The dotdashed line is the optical depth of a laboratory ice film deposited on a CsI window.

without the hydrate. This allows us to estimate the possible contribution of the water ice absorption wing due to scattering to the underlying continuum of the $3.47 \mu \mathrm{m}$ feature.

From column densities ratios from Table 1, the mantle/core volume ratio must be $r_{\mathrm{vol}} \geq 2$. We adopt 3 , as there is a region around these sources where exist some silicates without any ice mantles. This intermediate value is representative of the 2-5 range. The $a_{\max }$ is then adjusted to obtain the best line profile to compare with the observations, not taking into account the region between 3.2 and $3.8 \mu \mathrm{m}$, where the $3.47 \mu \mathrm{m}$ feature can appear. The results are shown in Fig. 5. The $a_{\max }$ upper limit needed to account for the water ice wing is in the $0.7-0.8 \mu \mathrm{m}$ range. Similar results were obtained by Smith et al. (1989). 
This analysis clearly shows that the scattering is needed to account for the water ice absorption wing but is not sufficient to produce the $3.47 \mu \mathrm{m}$ band. It also underlines that this band seems more extended than the width extracted using local continuum estimates. However, the local continuum approximation to extract this $3.47 \mu \mathrm{m}$ feature is still justified, provided that the continuum is adjusted from both sides of the 3.2 to $3.8 \mu \mathrm{m}$ range. This is different from the adopted local continuum of Brooke et al. (1996, 1999) and Chiar et al. (1996), which assumes the $3.47 \mu \mathrm{m}$ absorption band does not extend below $3.35 \mu \mathrm{m}$. The bulk of the optical depth can be explained with water ice mantles scattering effects, as already shown directly on astronomical spectra in the previous section. However, given the rapid increase of the optical depth in the $3.2-3.3 \mu \mathrm{m}$ wavelength region, together with the appearance in some sources of structure in the water ice profile with the onset of crystallinity, like in GL 2136, the continuum determination leaves some uncertainties that are difficult to appreciate and will be discussed in a next section. Using our scattering approach, the optical depth of the $3.47 \mu \mathrm{m}$ feature is therefore increased as compared to the one derived in the simple approximation used in Figs. 2 and 3 .

\section{The $3.47 \mu \mathrm{m}$ in the laboratory: Hydrate formation}

The residuals in the $3.47 \mu \mathrm{m}$ region of the optical depth plots of Fig. 5 (arrows) correspond to a few percent of the water ice depth (3\% to 5\%) and may find an explanation in the interactions taking place in the interstellar "matrices", as discussed below.

In the laboratory, co-deposition of water molecules with ammonia on a cold surface (at $10 \mathrm{~K}$ ) severely alters the resulting infrared spectrum. These two molecules interact to form an ammonia hydrate, and the infrared spectrum of such a mixture displays an additional feature at $3.47 \mu \mathrm{m}$, unexpected if these molecules are condensed separately under the same conditions. The formation of this hydrate is exemplified in the experiments shown in Fig. 6. $\mathrm{H}_{2} \mathrm{O}-\mathrm{NH}_{3}$ mixtures were deposited on a cold CsI substrate and their infrared spectra recorded. The amount of ammonia in the mixture was progressively increased with respect to water $\left(\mathrm{NH}_{3} / \mathrm{H}_{2} \mathrm{O}=0.015,0.03,0.07,0.2\right)$. All absorbance peaks are normalized to the strongest $\mathrm{H}_{2} \mathrm{O}$ vibrational mode at $3 \mu \mathrm{m}$. The pure $\mathrm{NH}_{3}$ ice spectrum is also presented. It is clear from these plots that a strong absorption arises at $2880 \mathrm{~cm}^{-1}(3.47 \mu \mathrm{m})$, which scales with the ammonia content. However, this band is not attributable to an ammonia fundamental mode, as can be easily seen by comparison with the pure $\mathrm{NH}_{3}$ ice absorption also presented in Fig. 6 .

When the absorbance of pure water ice is subtracted from that of a mixture containing $\mathrm{NH}_{3}$, with all spectra previously normalized to the water ice stretching modes, the spectra presented in Fig. 7 are obtained. The $\mathrm{NH}_{3}$ modes are easily identified on the figure. Surprisingly, some residual absorption from water ice modes $\left(3 v_{\mathrm{L}}, v_{2}\right.$ and $\left.v_{\mathrm{L}}\right)$ persist. This means that the oscillator strength of the water $\mathrm{OH}$ stretching mode has changed with respect to the other water modes, or, more correctly, that

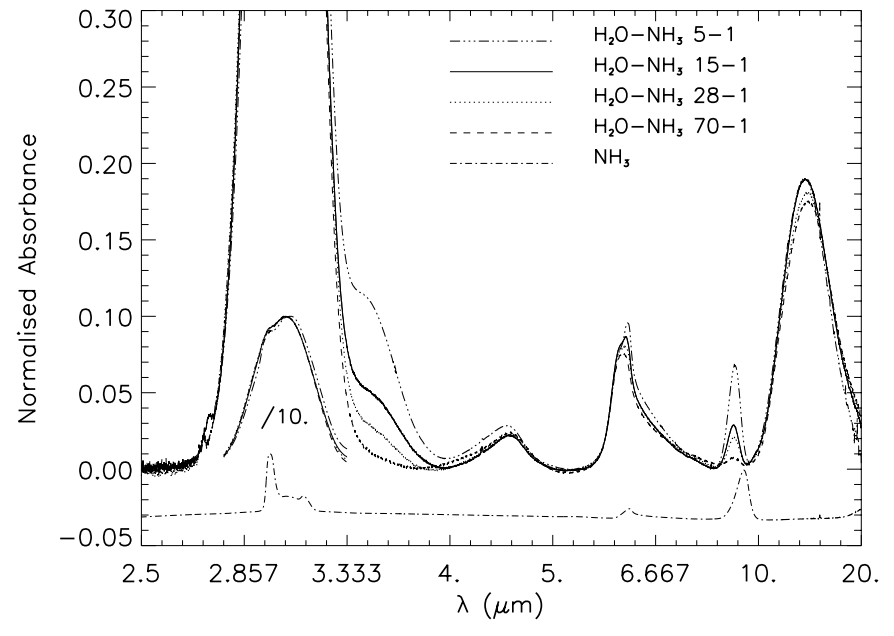

Fig. 6. Laboratory infrared absorbance spectra of $\mathrm{H}_{2} \mathrm{O} / \mathrm{NH}_{3}$ ice mixtures condensed at $10 \mathrm{~K}$. The spectra were normalized to the water ice content using the $\mathrm{OH}$ stretching mode around $3.1 \mu \mathrm{m} . \mathrm{NH}_{3} / \mathrm{H}_{2} \mathrm{O}$ proportions used are indicated on the top right of the spectra. A pure $\mathrm{NH}_{3}$ spectrum is displayed in the lower part for comparison. This normalization underlines the growth of a new infrared active mode at $3.47 \mu \mathrm{m}$ $\left(2880 \mathrm{~cm}^{-1}\right)$.

part of the $\mathrm{OH}$ groups are implied in another kind of interaction.

The $3.47 \mu \mathrm{m}\left(2880 \mathrm{~cm}^{-1}\right)$ mode is due to the interaction of the nitrogen atom of $\mathrm{NH}_{3}$ with an $\mathrm{OH}$ bond of the water molecule, forming an ammonia hydrate (Sill et al. 1981; Bertie et al. 1980). The nature of this mode explains both why it is broad, structureless, and intense, as we are seeing a perturbed water-OH absorption. To estimate its oscillator strength, we use the proportionality between the absorbance of the ammonia umbrella mode to the $3.47 \mu \mathrm{m}$ absorption. This is valid as long as $\mathrm{NH}_{3}$ is only an impurity in the water matrix, implying a single hydrate per ammonia molecule. By comparing the integrated cross section of the new mode to the $\mathrm{NH}_{3}$ umbrella mode, using classical integrated cross sections $\left(A_{9 \mu \mathrm{m}} \approx\right.$ $1.7-2 \times 10^{-17} \mathrm{~cm} \mathrm{~mol}^{-1}$, d'Hendecourt \& Allamandola 1986; Allamandola et al. 1988) we estimate that $A_{3.47 \mu \mathrm{m}} \approx 1.5$ $2.5 \times 10^{-16} \mathrm{~cm} \mathrm{~mol}^{-1}$. This mode possesses a very high integrated absorption cross section, which makes it a real tracer of the presence of hydrates in the ice mantles, and is typical of $\mathrm{OH}$ vibrations.

To extract the amorphous hydrate spectrum, we use two spectra with different amounts of ammonia. To first order the optical depth of the spectra can be written as:

$$
\begin{aligned}
& x \tau_{\text {Hydrate }}+(1-x) \tau_{\mathrm{H}_{2} \mathrm{O}} \\
& y \tau_{\text {Hydrate }}+(1-y) \tau_{\mathrm{H}_{2} \mathrm{O}}
\end{aligned}
$$

where $y>x$ represent the fraction of ammonia in the two mixtures. A first order hydrate spectrum can then be extracted by multiplying each spectrum by $(1-y)$ and $(1-x)$, respectively, and subtracting the second one from the first one.

When we add then about $7 \%$ of $\mathrm{NH}_{3}$ and the expected methanol contribution to the band to the fit parameters obtained in Fig. 5 (without the hydrate), we obtain the fits of Fig. 8. Note 


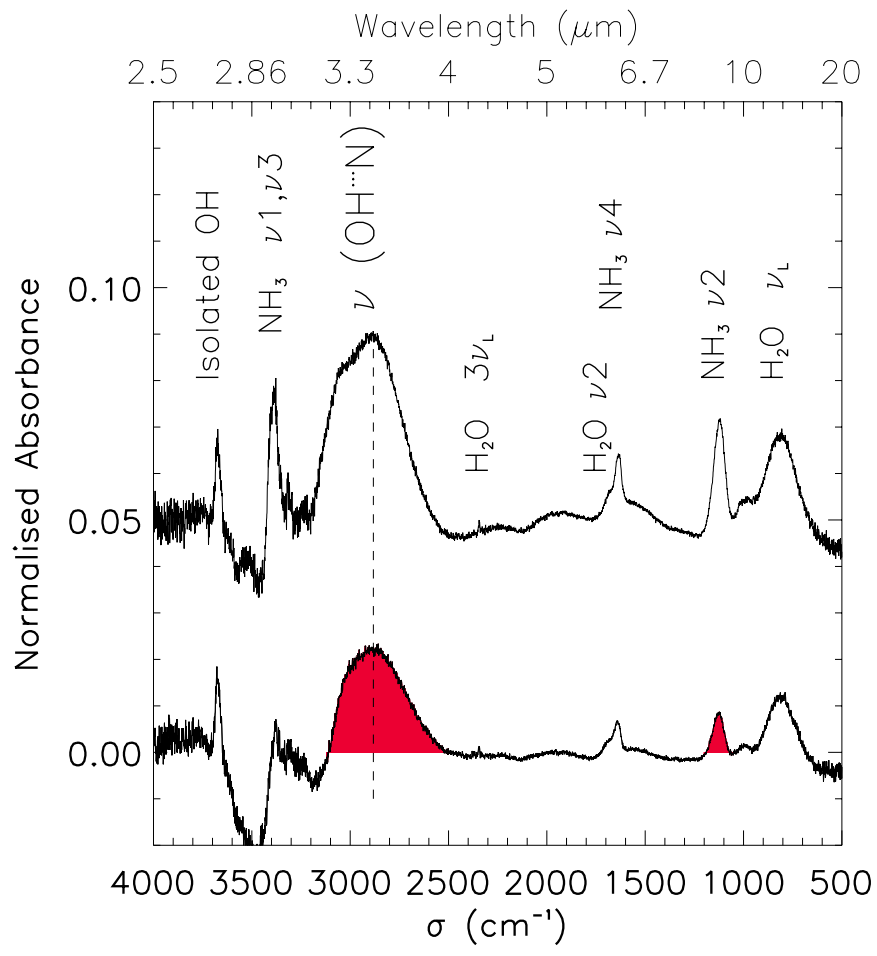

Fig. 7. Residual absorbance spectra obtained by subtracting $\mathrm{H}_{2} \mathrm{O}$ $\mathrm{NH}_{3}$ 15-1 to 70-1 (upper curve) and $\mathrm{H}_{2} \mathrm{O}-\mathrm{NH}_{3}$ 28-1 to 70-1 mixtures spectra after normalization on the water ice $\mathrm{OH}$ stretching mode. The various modes remaining after this subtraction are labeled above the curves. The prominent hydrate mode $(v(\mathrm{OH} \ldots \mathrm{N}))$ is the strongest mode appearing. The evaluation of its integrated absorbance value is performed using the ratio of the two lines in dark grey.

that the ammonia contribution is in the form of an hydrate at $30 \mathrm{~K}$. The amount of methanol relative to water in the fit of the observed $3.54 \mu \mathrm{m}$ absorption of GL 2136, is higher (7\% versus $5 \%$ ) than that derived previously by Brooke et al. (1999). This might reflect the use of scattering calculations in our determination of the water ice column density, which therefore appears less abundant than the direct determination on the $3 \mu \mathrm{m}$ absorption using simply the classical integrated absorbance technique, as already discussed in Dartois \& d'Hendecourt (2001).

Another approach consists of subtracting the ice mantle fit without ammonia from the interstellar absorption and compare the residual to laboratory experiments directly in optical depth. Note that the total optical depth in the $3.47 \mu \mathrm{m}$ band is higher than that extracted using a local continuum which goes through the $3.35 \mu \mathrm{m}$ region. To be consistent, we proceed in the same way for the experiment, where instead of drawing an arbitrary local continuum on the spectrum, we subtract the normalized optical depth of a spectrum recorded with a lower $\mathrm{NH}_{3} / \mathrm{H}_{2} \mathrm{O}$ ratio. The comparison are presented in Fig. 9. We also overplot (long dashed line) the difference between the Mie scattering best fits using only water ice contribution and using water ice plus hydrate plus methanol mixture.

The ratio of the $3.25 \mu \mathrm{m}$ to $3.47 \mu \mathrm{m}$ band in the laboratory spectra varies with the amount of ammonia and is more pronounced in the crystalline case (GL 2136 case). This is not the appearance of a new band around $3.25 \mu \mathrm{m}$ but a difference
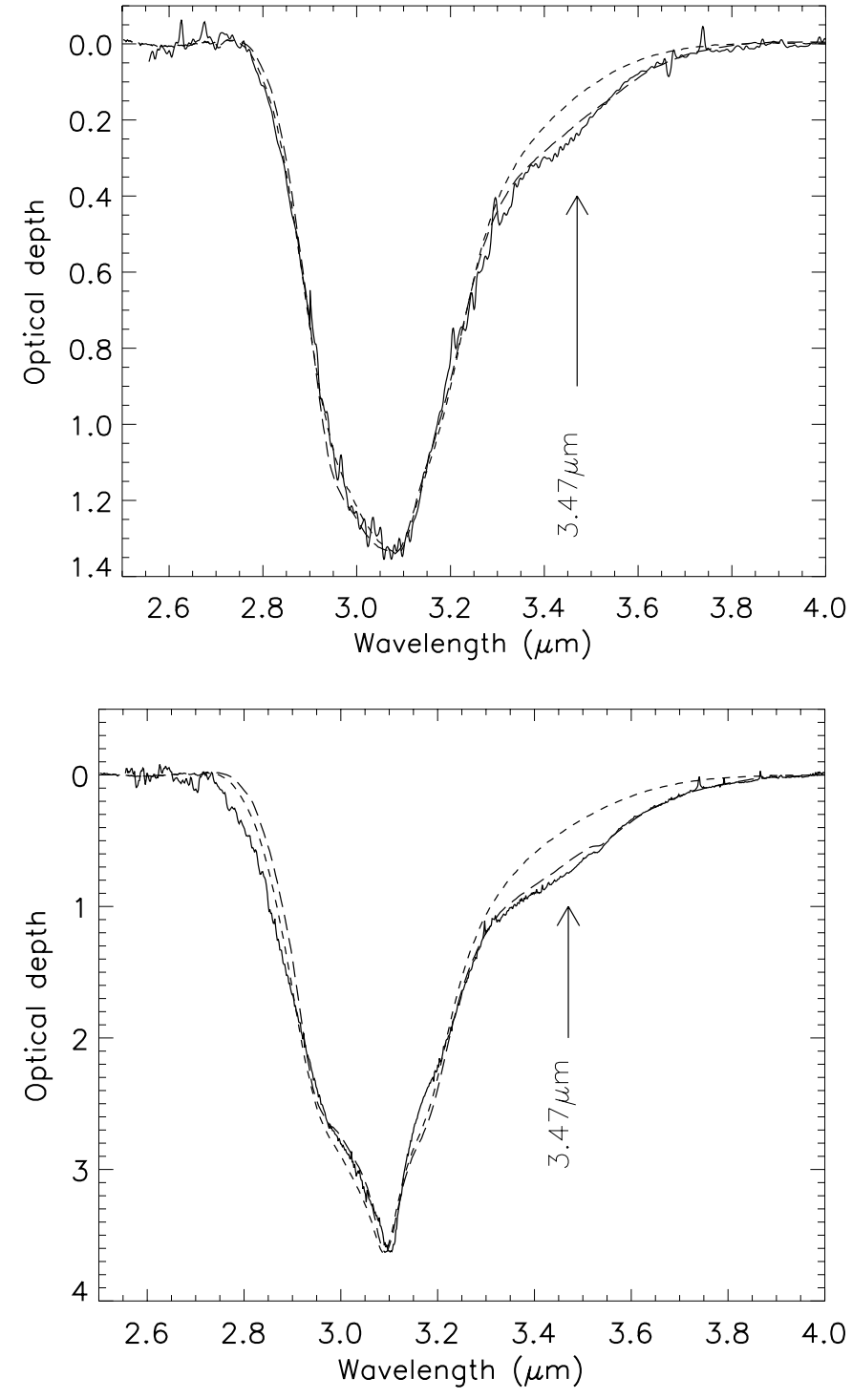

Fig. 8. Optical depth spectra of GL 989 (upper panel) and GL 2136 (lower panel) in the $L$ band. The short dashed line represents the silicate core-ice mantle fit using laboratory data and parameters described in Table 2, and presented in Fig. 5. The long dashed line use the same parameters, except that $7 \%$ of hydrate was added to produce the $3.47 \mu \mathrm{m}$ absorption.

in the $\mathrm{H}_{2} \mathrm{O}$ crystalline profile resulting from slightly different molecular arrangement when ammonia is embedded or not in the ice matrix. It means that in the astronomical case, the local extraction in this wavelength region is much more critical than for e.g. the $4.67 \mu \mathrm{m} \mathrm{CO}$ band, as it might reflect the phase changes of the crystalline $\mathrm{H}_{2} \mathrm{O}$. It would explain why this $3.25 \mu \mathrm{m}$ feature is not correlated with the water ice optical depth (Brooke et al. 1999), but is only present in the spectra of lines of sight where evolved ice mantles are present.

This $3.47 \mu \mathrm{m}$ band must appear as long as some $\mathrm{NH}_{3}$ is embedded in the water ice mantle, which makes it a stringent and powerful constraint on the maximum possible amount of $\mathrm{NH}_{3}$ in the observed interstellar ice mantles, i.e. equal or less than $7 \%$, even if one invokes other mechanisms to produce part of this extinction. 

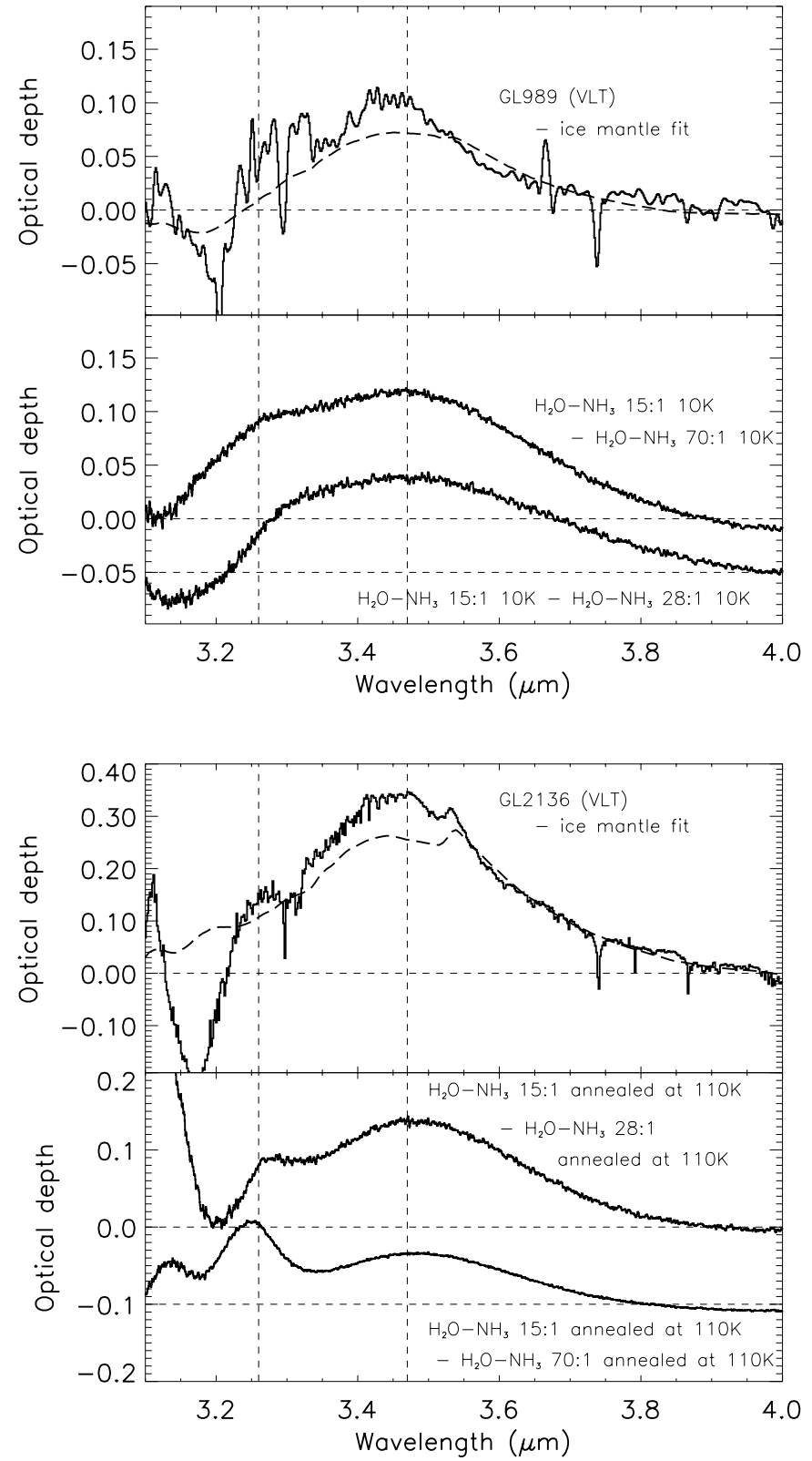

Fig. 9. Residue obtained by subtracting the best fit without hydrate presented in Fig. 8 to the VLT data. The long dashed superimposed line represent the difference between the fits obtained in Fig. 8 with hydrate + methanol + water ice and with pure ice. Comparisons with $\mathrm{NH}_{3} / \mathrm{H}_{2} \mathrm{O}$ ice films laboratory data residues are displayed for comparison of the profiles. The laboratory ammonia hydrate residues are obtained by subtracting a spectrum of an ice film with almost no ammonia. The expected positions of the so-called 3.25 and $3.47 \mu \mathrm{m}$ bands are given by vertical dashed lines. Note that the VLT residue is affected by a strong and sharp telluric methane absorption band around $3.3 \mu \mathrm{m}$.

\section{Discussion of the $3.47 \mu \mathrm{m}$ mode}

We suggest that the $3.47 \mu \mathrm{m}$ absorption band observed toward many astronomical lines of sight is partly due to the presence of mixed ammonia in interaction with the water ice mantle. This would be the direct consequence of the dominant presence of solid water in grain mantles. Few infrared absorptions arising from simple molecules, with such a broad and intense mode can take place at that particular wavelength.

Alternatively, $\mathrm{NH}_{4}^{+}$has been suggested to contribute to part of this mode (Schutte et al. 2002). $\mathrm{NH}_{4}^{+}$is also believed to produce some extinction in the $6.85 \mu \mathrm{m}$ absorption observed toward many interstellar sources (Keane et al. 2001). It is often linked to the presence of the so-called " $\mathrm{XCN}$ " feature, an absorption at $4.62 \mu \mathrm{m}$ attributed to the $\mathrm{OCN}^{-}$ion (Grim \& Greenberg 1987; Grim et al. 1989; Schutte \& Greenberg 1997; Demyk et al. 1998; Whittet et al. 2001; Hudson et al. 2001; Novozamsky et al. 2001), one of the $\mathrm{NH}_{4}^{+}$counter-ions, to preserve a relative neutrality in the ice mantle.

GL 989 and GL 2136 are interesting sources chosen for mantle composition comparisons because the former does not show any strong $\mathrm{OCN}^{-}$feature but the second does, whereas they both exhibit a $3.47 \mu \mathrm{m}$ feature whose relative optical depth is equivalent with respect to the main $\mathrm{H}_{2} \mathrm{O}$ ice component. This shows that there is no direct link between this $3.47 \mu \mathrm{m}$ band and the $\mathrm{OCN}^{-}$feature, which would be expected if $\mathrm{NH}_{4}^{+}$is the unique positive ion present in the mantle.

The circumstellar material in these sources differs in its evolutionary stage, the ice being dominated by the crystalline state in GL 2136 and amorphous one in GL 989. The $\mathrm{OCN}^{-}$ion is generally the sign of chemically evolved sources. To allow an acid-base reaction or a photo-chemically assisted reaction to produce this ion in the ice mantle, the presence of nitrogen is therefore a prerequisite.

The occurrence of the $3.47 \mu \mathrm{m}$ absorption feature in sources ranging from intermediate (Thi et al. 2002) to high mass objects, and in sources presenting evolved features and high temperature (crystalline) ice as well as amorphous features implies that the involved molecules must be present at any step of the ice mantle evolution, being a rather non-volatile and ubiquitous ice component.

\section{Implications on the global spectrum: The $10 \mu \mathrm{m}$ range}

\section{1. $\mathrm{CH}_{3} \mathrm{OH}$ ice on a silicate film}

The molecules responsible for the $3.47 \mu \mathrm{m}$ and $3.54 \mu \mathrm{m}$ features, i.e. $\mathrm{NH}_{3}$ and $\mathrm{CH}_{3} \mathrm{OH}$ possess fundamental absorptions at other frequencies. This is evidenced in Fig. 6 for $\mathrm{NH}_{3}$ whose strongest mode lies at $\sim 1115 \mathrm{~cm}^{-1}(8.96 \mu \mathrm{m})$ when embedded in an $\mathrm{H}_{2} \mathrm{O}$ polar matrix. In the methanol case, the strong CO elongation mode $\left(v_{8}\right)$ falls at $\sim 1017-1026 \mathrm{~cm}^{-1}(9.75-$ $9.83 \mu \mathrm{m})$, depending on the mixture involved. However, in astronomical spectra these methanol and ammonia "fingerprints" remain hidden.

To illustrate and validate this idea within the context of a laboratory experiment, we have condensed a methanol ice film on top of a silicate film, to measure the resultant transmittance. We assess in this way the observability of the features in a realistic case. The silicate film was first deposited on a cold CsI window from a pure enstatite mineral by heating the sample with an ionic beam in an evacuated system. The CsI window was placed in the system and a $5000 \AA$ thick amorphous silicate film was then condensed on the CsI window. Residual 
crystalline absorptions do exist in the film, but the global spectrum is amorphous. The CsI window was then placed in our cryostat and cooled down to $10 \mathrm{~K}$. Another pure CsI window was placed in the same cryostat. After recording reference and cold silicate transmission spectra, methanol was then condensed on each window in order to reach an optical depth of about 0.1 in the $\mathrm{OH}$ stretching mode region corresponding to a column density of about $2 \times 10^{17} \mathrm{~cm}^{-2}$, typical of the maximum amount of $\mathrm{CH}_{3} \mathrm{OH}$ present in high mass lines of sight such as W33 A and RAFGL7009 (Dartois et al. 1999). After CsI baseline correction, these spectra are presented in Fig. 10.

A closer view of the silicate $\mathrm{SiO}$ stretching region, which includes the $v_{8}$ mode of $\mathrm{CH}_{3} \mathrm{OH}$, is presented in the lower panel of Fig. 10. The upper spectrum (A) is the silicate absorption at $10 \mathrm{~K}$ whereas the lower one (B) includes the methanol $v_{8}$ absorption. This mode is thus hardly observable directly in the laboratory data. It illustrates rather well the difficulty to extract these strong modes, a priori, from a silicate absorption band whose profile is not accurately known. It also shows that silicates only slightly crystalline can display large bands, whose width is typical of the $\mathrm{NH}_{3}$ umbrella mode embedded in water ice, in the shoulder of the $\mathrm{SiO}$ stretching mode absorption region, which are therefore difficult to analyze with a polynomial local continuum approximation.

When observing astronomical targets, the extraction is even worse as the spectral signal-to-noise ratios decrease drastically in the low flux part of the silicate band. In summary, if one reviews the observable fundamental methanol modes in an astronomical context, the $\mathrm{OH}$ absorption as well as the modes on the blue side of the $3.54 \mu \mathrm{m}$ absorption, are mixed and hidden within the deep water ice feature (see Fig. 7 of Dartois et al. 1999). The $6.85 \mu \mathrm{m}$ absorptions $\left(\mathrm{CH}_{3}\right.$ deformations and $\mathrm{OH}$ bending) fall in a crowded infrared spectral region, where many absorptions from aliphatic molecules can provide some extinction, preventing an unambiguous assignment. An extraction of the $\mathrm{CO}$ mode around $9.7 \mu \mathrm{m}$ implies that we must know a priori the exact structure of the interstellar silicate band, remembering that the contrast is actually very low, as seen in Fig. 10. Therefore we expect to detect methanol in astronomical sources mainly via its $v_{3}$ mode at $\sim 3.54 \mu \mathrm{m}$. Indeed, in GL 2136 and GL 989, as well as W33 A, NGC 7538 IRS9 (Allamandola et al. 1992) and AFGL 7009 (Dartois et al. 1999), a rather high abundance of solid methanol was primarily detected via this mode and not via the stronger $v_{8}$ mode.

\subsection{The contrast problem for the $\mathrm{NH}_{3}-\mathrm{CH}_{3} \mathrm{OH}$ 9 to $10 \mu \mathrm{m}$ modes}

In order to test the compatibility with the short wavelength feature assignment presented above, we now focus on the inverse problem for $\mathrm{NH}_{3}$ and $\mathrm{CH}_{3} \mathrm{OH}$ modes at $\sim 10 \mu \mathrm{m}$. We need first to extract an accurate as possible silicate profile in each infrared source spectrum. We searched in the ISO database for high mass infrared sources with similar spectral energy distribution and silicate profiles as those encountered in our sources, but possessing lower ice column densities. These sources are then considered as reference sources or "standards". We extract

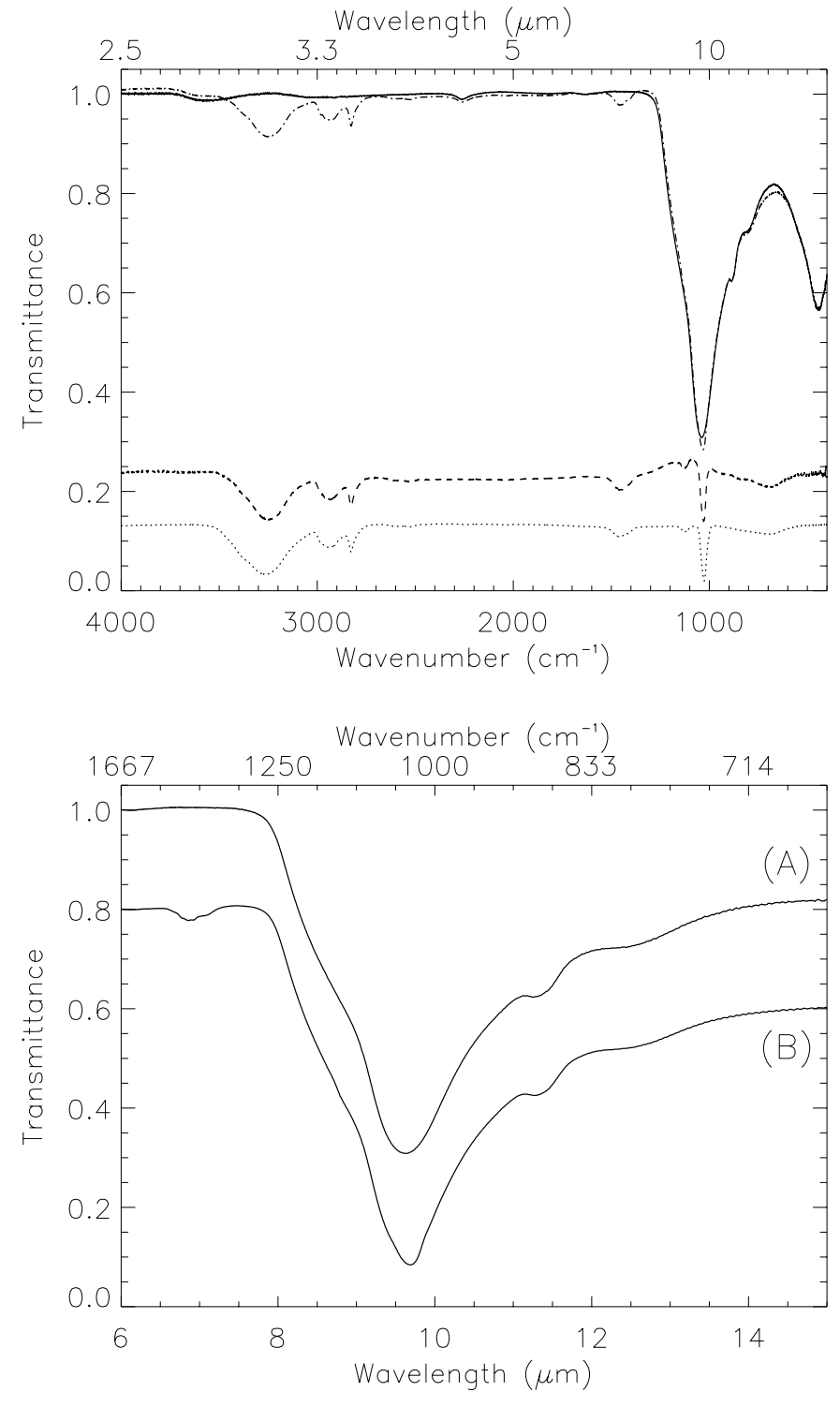

Fig. 10. Laboratory spectra of methanol deposited on an amorphous enstatite film. Upper panel: (i) $5000 \AA$ thick enstatite film at $10 \mathrm{~K}$ (full line), (ii) same film + methanol (dot-dashed line), (iii) extracted methanol spectrum (dashed line) obtained by dividing (ii) by (i), shifted for better clarity and (iv) pure methanol film deposited on a CsI window (dotted line). Lower panel: close-up of the silicate $\mathrm{SiO}$ stretching mode region of the enstatite (A) and enstatite + methanol (B) spectra from the upper panel. The low contrast of the $v 8$ methanol mode around $9.7 \mu \mathrm{m}$ resulting from the onset of the silicates absorption renders its detection problematic if the silicate profile is not perfectly known a priori.

the silicate optical depth for each source, taking care to use at least two "standards" for each science target. We then adjust the silicate depth of the reference sources to our studied sources, in such a way that the optical depth of the reference never exceed the source one in the high depth region of the spectrum. In this way, we search for the presence of any additional ice contribution. This approach is presented in Fig. 11 for GL 2136 and GL 989 and is much safer than the local continuum approximation used by e.g. Gibb et al. (2001) as will be discussed below. 

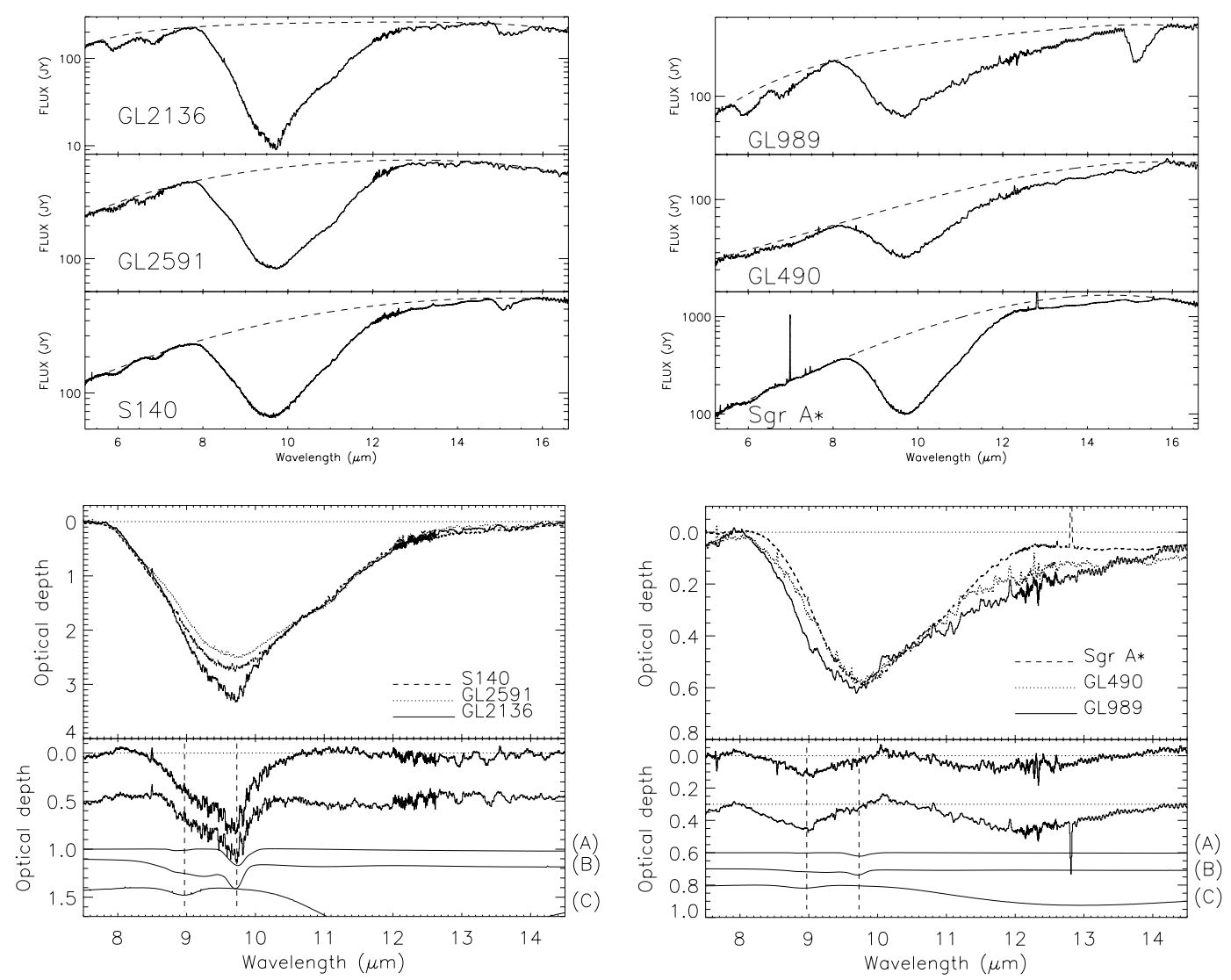

Fig. 11. Upper left panel: ISO SWS01 spectra of the embedded massive protostars GL 2136, GL2591 and S140 in the silicate stretching mode absorption region and their respective adopted local continua (dashed lines). Lower panel, upper part: optical depth spectra of the above sources normalized using the wing of the GL 2136 silicate absorption. Lower left panel, lower part: residual optical depth after subtraction of the GL 2136 silicate absorption by the GL 2591 and S 140 ones normalized as explained above. The residuals are then compared to ice spectra of (A) $\mathrm{CH}_{3} \mathrm{OH}$, (B) $\mathrm{CH}_{3} \mathrm{OH} / \mathrm{NH}_{3}$ (1:1) and (C) $\mathrm{H}_{2} \mathrm{O} / \mathrm{NH}_{3}$ (1:15) spectra. The vertical dashed lines indicates the positions of the strongest $\mathrm{NH}_{3}$ $(\approx 9 \mu \mathrm{m})$ and $\mathrm{CH}_{3} \mathrm{OH}(\approx 9.7 \mu \mathrm{m})$ absorptions. Right panels. Same as in left panels for GL 989 using GL490 and Sgr A* as "silicates" references. See text for more details.

It assumes that the chosen sources can be classified with similar radiative transfer properties for the silicate bands.

\subsubsection{GL 2136}

The normalization for GL 2136 using two silicate "standards" (see Fig. 11) demonstrates that there exist indeed an excess absorption around $9.7 \mu \mathrm{m}$ due to the methanol molecule, already identified with the mode at $3.54 \mu \mathrm{m}$, and previously seen using ground based observations by Skinner et al. (1992). Based on the optical depth of the short wavelength feature, we over-plot the expected contribution to this excess using a laboratory spectrum of pure methanol recorded at $10 \mathrm{~K}$ (spectrum labeled " $\mathrm{A}$ " in the lower panel of Fig. 11). The match is perfect for this line. A small excess around $9 \mu \mathrm{m}$ still exists, which fits with the presence of ammonia, as shown with the "B" spectrum, which represents a spectrum obtained with the same amount of methanol and ammonia, and normalized to the observed methanol feature. The last spectrum ("C") is a view of the $\mathrm{H}_{2} \mathrm{O}: \mathrm{NH}_{3}=15: 1$ mixture already presented in Fig. 7. Note that the very broad librational mode of $\mathrm{H}_{2} \mathrm{O}$ ice has been partially reduced in the silicate extraction process, showing again the difficulty of broad band extraction on imperfectly known continua.

The $3.47 \mu \mathrm{m}$ absorption, identified with ammonia hydrate, is compatible with the spectrum in the $10 \mu \mathrm{m}$ region. However, since the optical depth of the GL 2136 silicate feature exceeds 3 , the precise absolute optical depth in the $\mathrm{NH}_{3}$ region is particularly difficult to estimate. Furthermore, the $9 \mu \mathrm{m}$ feature of ammonia is quite broad $(0.6 \mu \mathrm{m})$ and falls in the wing of the silicate absorption, which complicates the extraction task. This is clearly an example of the difficulties and limitations of the continuum extraction imposed by the silicate band. In particular, the determination of the offset which defines the zero level in the reduction schemes of true astronomical data can severely affect the profiles at low flux levels, in addition to the intrinsic silicate profile determination. The $\mathrm{NH}_{3}$ extraction process should therefore be looked with care when molecules firmly detected in other regions of the spectrum, such as $\mathrm{CH}_{3} \mathrm{OH}$ who does possess adjacent absorption features, do not show up in the spectrum. This is especially the case in sources where we already know they should possess relatively high methanol abundances (e.g. W33 A, AFGL 7009 or NGC 7538 IRS9, see Gibb et al. 2000; Gürtler et al. 2002). 


\subsubsection{GL 989}

We proceed in the same way for GL 989. The silicate standards chosen on the basis of the similarity of the spectral energy distribution (hoping the radiative transfer is therefore similar) are in this case less good to reproduce the expected pure silicate profile. Sgr A* might especially be a non adapted example of a silicate band due to the intrinsic emission which narrows the profile. A possible $9.7 \mu \mathrm{m}$ methanol contribution is not clear, contrary to GL 2136. Still the excess observed in the $9 \mu \mathrm{m}$ region is compatible with the assignment of the $3.47 \mu \mathrm{m}$ feature to the ammonia hydrate formation. The "A" through " $\mathrm{C}$ " labeled curves have the same meaning as for GL 2136, and have just been normalized to the GL 989 methanol $3.54 \mu$ m observed band accordingly.

\section{Astronomical issue}

The determination of the nitrogen content in ices is of prime importance to understand the subsequent evolution of ices subjected to irradiation and/or ion bombardment. Nitrogencontaining species are hardly identified up to now. The expected more abundant species after the very volatile and almost unobservable nitrogen molecule is ammonia. A tentative detection of the NH stretching mode at $2.97 \mu \mathrm{m}$ was claimed and discarded by Graham et al. (1991, 1998), whereas Whittet discussed its observability in HH100 (Whittet et al. 1996) if ammonia represents a low percentage of the molecular ice content. The strongest mode of $\mathrm{NH}_{3}$ falling around $9 \mu \mathrm{m}$ is searched for by many authors (Lacy et al. 1998; Gibb et al. 2001; Chiar et al. 2000; Gurtler et al. 2002). In this case, as we have shown, the extraction of an ice band in the silicate absorption wing is highly approximate for such a broad feature of about $60 \mathrm{~cm}^{-1}$ width.

In the gas phase, the fractional abundance of ammonia molecules relative to $\mathrm{H}_{2}$ in hot cores is relatively high $\left(10^{-5}\right.$ to $10^{-6}$ e.g. Cesaroni et al. 1994) whereas in protostellar cores it is much lower $\left(10^{-7}\right.$ to $10^{-9}$ e.g. Shah \& Wootten 2001; Tiné et al. $2001)$ although highly deuterated $\left(10^{-1}>\mathrm{NH}_{2} \mathrm{D} / \mathrm{NH}_{3}>10^{-2}\right)$. The high fractionation of $\mathrm{NH}_{3}$ can be explained by gas phase chemistry plus grain surfaces acting as a depletion mechanism.

The nitrogen abundances derived from our interpretation involving hydrate formation to account for the broad $3.47 \mu \mathrm{m}$ absorption band are compatible with at most $7 \%$ of solid $\mathrm{NH}_{3}$ respective to $\mathrm{H}_{2} \mathrm{O}$, a similar value for both sources. According to chemistry models involving gas and grain interactions (e.g. d'Hendecourt et al. 1985; Hasegawa et al. 1992), the process of formation of simple hydrogenated molecules $\left(\mathrm{H}_{2} \mathrm{O}, \mathrm{NH}_{3}\right.$ $\mathrm{CH}_{4}$ ) via hydrogen atoms on grain surfaces is identical for $\mathrm{NH}_{3}$ and $\mathrm{H}_{2} \mathrm{O}$. Thus with a cosmic $\mathrm{N} / \mathrm{O}$ ratio about 0.15 (e.g. Snow \& Witt 1996), one would expect a $15 \%$ abundance of this molecule with respect to $\mathrm{H}_{2} \mathrm{O}$.

With this amount of ammonia, nitrogen is not highly underabundant as compared to other molecules in grain mantles. The possibility to form $\mathrm{N}_{2}$ molecules has been emphasized in grain models. Unfortunately $\mathrm{N}_{2}$ is undetectable at these wavelengths by direct methods. We note that this abundance is enough to explain the high abundance of $\mathrm{NH}_{3}$ in the gas phase observations, as in the sources discussed here, a $1 \%$ abundance with respect to water ice implies absolute abundances of the order of $10^{-6}$ to $10^{-7}$ with respect to $\mathrm{H}_{2}$.

In addition, the presence of small amounts of ammonia in ice mantles is expected on the basis of the observation of other features $\left(\mathrm{OCN}^{-}, \mathrm{NH}_{4}^{+}\right)$. Indeed, a classical scheme for the evolution of the grain mantles, as monitored in the laboratory experiments, is the production via photochemical reactions involving $\mathrm{CO}$ and $\mathrm{NH}_{3}$ of $\mathrm{HNCO}+\mathrm{NH}_{3}$, followed by a rapid proton exchange to form $\mathrm{OCN}^{-}-\mathrm{NH}_{4}^{+}$(Hudson et al. 2001 and reference therein, Demyk et al. 1998). Similar reaction products can be obtained with ice mixtures containing $\mathrm{N}_{2}$ and $\mathrm{H}_{2} \mathrm{O}$ (water providing the necessary $\mathrm{H}$ atoms to produce $\mathrm{HNCO}$ ), but using proton bombardments, as UV photolysis with typically $10 \mathrm{eV}$ photons is not efficient enough to dissociate the stable $\mathrm{N}_{2}$ diatomic molecule.

Whatever the scheme implied in the formation route of $\mathrm{OCN}^{-}$(ion bombardment, surface reactions, UV photolysis), it probably involves $\mathrm{NH}_{4}^{+}$, and therefore $\mathrm{NH}_{3}$.

\section{Conclusion}

The $3.47 \mu \mathrm{m}$ feature is identified with the presence of a few percent of ammonia in ices, where ammonia is intimately linked with $\mathrm{H}_{2} \mathrm{O}$. The formation of such an ammonia hydrate feature is a natural consequence of the water ice dominated mantle composition. This band is due to a perturbed water $\mathrm{OH}$ stretching mode, which explains the broadness of this feature, shifted by the interaction with the nitrogen atom of the ammonia molecule. This feature seems ubiquitous in grain mantles and globally scales with the water ice amount. It is present at all evolution stages of the protostars, as we detect it in amorphous and crystalline ice mantles surrounding GL 989 and GL 2136, two differently evolved sources.

This $3.47 \mu \mathrm{m}$ feature allows to observe (or put constraints on) the solid $\mathrm{NH}_{3}$ column densities as compared to the main water ice constituent of grain mantles, as the main other transitions of solid ammonia are hidden in the rich and complex spectra of protostars. If the absorption in this $3.47 \mu \mathrm{m}$ feature is entirely due to the formation of an ammonia hydrate, ammonia represents at most $7 \%$ of the water ice mantle content.

In relation with the already identified $\mathrm{NH}_{4}^{+}$ion and one of its counter-ions $\mathrm{OCN}^{-}$in evolved sources, we propose an evolutionary sequence for ices in which the initial $\mathrm{NH}_{3}$ is partly consumed to produce more complex molecules such as HNCO, and then $\mathrm{OCN}^{-}-\mathrm{NH}_{4}^{+}$. Eventually this may later on lead to the formation of urea when the mantle is subjected to heating processes during the evolution of the parent cloud.

Finally, we stress that the identification of a solid state molecule must now consider the fundamental vibrational spectra from 2 to $25 \mu \mathrm{m}$ when available from the ISO database. In wavelength windows accessible from ground based astronomy, this can be examined with much better sensitivity. VLT-ISAAC observations have thus allowed to examine and interpret the profile of the $3.47 \mu \mathrm{m}$ feature with a high precision, a band whose optical depth is only 0.04 in the case of GL 989, but which may trace intimate physical association of molecules in the solid state. 
Acknowledgements. We wish to thank the VLT team operating the ISAAC facility on UT1. We are specifically grateful to Chris Lidman and Olivier Marco for many helpful comments on site during observations.

\section{References}

Allamandola, L. J., Sandford, S. A., Tielens, A. G. G. M., \& Herbst, T. M. 1992, ApJ, 399, 134

Allamandola, L. J., Sandford, S. A., \& Valero, G. J. 1988, Icarus, 76, 225

Bertie, J. E., \& Morrison, M. M. 1980, J. Chem. Phys., 73, 4832

Brooke, T. Y., Sellgren, K., \& Geballe, T. R. 1999, ApJ, 517, 883

Brooke, T. Y., Sellgren, K., \& Smith, R. G. 1996, ApJ, 459, 209

Capps, R. W., Gillett, F. C., \& Knacke, R. F. 1978, ApJ, 226, 863

Cesaroni, R., Churchwell, E., Hofner, P., Walmsley, C. M., \& Kurtz, S. 1994, A\&A, 288, 903

Chiar, J. E., Tielens, A. G. G. M., Whittet, D. C. B., et al. 2000, ApJ, 537, 749

Chiar, J. E., Adamson, A. J., \& Whittet, D. C. B. 1996, ApJ, 472, 665

Cox, P. 1989, A\&A, 225, L1

Dartois, E., \& d'Hendecourt, L. 2001, A\&A, 365, 144

Dartois, E., Schutte, W., Geballe, T. R., et al. 1999, A\&A, 342, L32

Demyk, K., Dartois, E., D’Hendecourt, L., et al. 1998, A\&A, 339, 553

van Dishoeck, E. F., Dartois, E., Thi, W. F., et al. 2002, The Origins of Stars and Planets: The VLT View, ed. J. Alves, \& M. McCaughrean (Springer Verlag)

Draine, B. T., \& Lee, H. M. 1984, ApJ, 285, 89

Ehrenfreund, P., Dartois, E., Demyk, K., \& D'Hendecourt, L. 1998, A\&A, 339, L17

Geballe, T. R. 1986, A\&A, 162, 248

Gibb, E. L., Whittet, D. C. B., \& Chiar, J. E. 2001, ApJ, 558, 702

de Graauw, T., et al. 1996, A\&A, 315, L345

Graham, J. A. 1998, ApJ, 492, 213

Graham, J. A., \& Chen, W. P. 1991, AJ, 102, 1405

Grim, R. J. A., Greenberg, J. M., de Groot, M. S., et al. 1989, A\&AS, 78,161

Grim, R. J. A., \& Greenberg, J. M. 1987, ApJ, 321, L91

Guertler, J., Henning, T., Koempe, C., et al. 1996, A\&A, 315, L189

Gurtler, J., Klaas, U., Henning, Th., et al. 2002, A\&A, 390, 1075

Hasegawa, T. I., Herbst, E., \& Leung, C. M. 1992, ApJS, 82, 167

d'Hendecourt, L., \& Dartois, E. 2001, Spectrochimica Acta, 57, 669

d'Hendecourt, L., et al. 1996, A\&A, 315, L365

d'Hendecourt, L. B., \& Jourdain de Muizon, M. 1989, A\&A, 223, L5

d'hendecourt, L. B., \& Allamandola, L. J. 1986, A\&AS, 64, 453

d'Hendecourt, L. B., Allamandola, L. J., \& Greenberg, J. M. 1985, A\&A, 152, 130
Henning, T., Pfau, W., \& Altenhoff, W. J. 1990, A\&A, 227, 542

Hudson, R. L., Moore, M. H., \& Gerakines, P. A. 2001, ApJ, 550, 1140

Kastner, J. H., Weintraub, D. A., Snell, R. L., et al. 1994, ApJ, 425, 695

Keane, J. V., Tielens, A. G. G. M., Boogert, A. C. A., Schutte, W. A., \& Whittet, D. C. B. 2001, A\&A, 376, 254

Lacy, J. H., Faraji, H., Sandford, S. A., \& Allamandola, L. J. 1998, ApJ, 501, L105

Lacy, J. H., Baas, F., Allamandola, L. J., van de Bult, C. E. P., et al. 1984, ApJ, 276, 533

Leger, A., Klein, J., de Cheveigne, S., et al. 1979, A\&A, 79, 256

Mathis, J. S., Rumpl, W., \& Nordsieck, K. H. 1977, ApJ, 217, 425

Merrill, K. M., Russell, R. W., \& Soifer, B. T. 1976, ApJ, 207, 763

Novozamsky, J. H., Schutte, W. A., \& Keane, J. V. 2001, A\&A, 379, 588

Omont, A., Forveille, T., Moseley, S. H., Glaccum, W. J., et al. 1990, ApJ, 355, L27

Pendleton, Y. J., Sandford, S. A., Allamandola, L. J., Tielens, A. G. G. M., \& Sellgren, K. 1994, ApJ, 437, 683

Roche, P. F., \& Aitken, D. K. 1984, MNRAS, 209, 33P

Roueff, E., Tiné, S., Coudert, L. H., et al. 2000, A\&A, 354, L63

Sandford, S. A., Allamandola, L. J., Tielens, A. G. G. M., et al. 1991, ApJ, 371, 607

Schutte, W. A., et al. 2002, in preparation

Schutte, W. A., \& Greenberg, J. M. 1997, A\&A, 317, L43

Shah, R. Y., \& Wootten, A. 2001, ApJ, 554, 933

Smith, R. G., Sellgren, K., \& Tokunaga, A. T. 1989, ApJ, 344, 413

Smith, R. G., Sellgren, K., \& Tokunaga, A. T. 1988, ApJ, 334, 209

Sill, G., Fink, U., \& Ferraro, J. R. 1981, J. Chem. Phys., 74, 997

Skinner, C. J., Tielens, A. G. G. M., Barlow, M. J., \& Justtanont, K. 1992, ApJ, 399, L79

Snow, T. P., \& Witt, A. N. 1996, ApJ, 468, L65

Soifer, B. T., Puetter, R. C., Russell, R. W., et al. 1979, ApJ, 232, L53

Thi, et al. 2002, in preparation

Tielens, A. G. G. M., Tokunaga, A. T., Geballe, T. R., \& Baas, F. 1991, ApJ, 381, 181

Tielens, A. G. G. M., \& Hagen, W. 1982, A\&A, 114, 245

Tiné, S., Roueff, E., Falgarone, E., Gerin, M., \& Pineau des Forêts, G. 2000, A\&A, 356, 1039

Willner, S. P., et al. 1982, ApJ, 253, 174

Whittet, D. C. B., Pendleton, Y. J., Gibb, E. L., et al. 2001, ApJ, 550, 793

Whittet, D. C. B., et al. 1996, ApJ, 458, 363

Whittet, D. C. B., \& Duley, W. W. 1991, A\&A Rev., 2, 167

Whittet, D. C. B., McFadzean, A. D., \& Longmore, A. J. 1985 , MNRAS, 216, 45P 